\title{
Increasing the Resolution of an Integrated Adaptive Spike Coded Sensor to Digital Conversion Neuro-Circuit by an Enhanced Place Coding Layer
}

\author{
Abhaya Chandra Kammara, Andreas König \\ Technische Universität Kaiserslautern, 67663 Kaiserslautern, \\ E-Mail: \{abhay,koenig\}@eit.uni-kl.de
}

\section{Abstract}

With the rapid advance of modern micro- and nanoelectronics, devices become more vulnerable to failure as well as to noise. The need for fault tolerant devices and circuits, that can compensate degradation and failure by adaptation, are inherently immune to noise, and compute at low-power gives new incentive to the motivation and goals of neuromorphic computing again. In particular, the realization of sensor signal conditioning and conversion by neural circuits coexisting with potentially large digital circuits on the same substrate is an attractive field for these techniques. In our work, for the case of a selected magnetic sensor (AMR), a spike-coded circuit employing principles from the auditory system is advanced, that already meets several of the goals mentioned above. Now we achieve 6.3 bit ADC with a sampling rate of $1 \mathrm{MHz}$ by using Rank Order Coding and a simple look-up table for decoding. The increase of resolution and linearity as well as introduction of adaptivity will be regarded next before considering microelectronic implementation.

\section{Introduction}

Sensor signal conditioning has seen many valuable approaches to solve different problems, with conventional techniques focusing on high resolution, high sampling rate and low power. These make use of amplitude coding which is highly susceptible to noise and vulnerable to soft and hard errors. Even the 
reconfigurable systems with programmable features like resolution, sampling rate etc still make use of the noisy amplitude coding. Time coded signals have become more popular recently both in research and industry [7]. We can also find biologically inspired time based approaches dealing with the ADC problem [6] which are robust and consume low power. These systems offer the promise of increased robustness and reduced vulnerability to noise and device deficiencies and defects as compared to the amplitude based systems. However they are not yet programmable, reconfigurable or adaptive. In our work, we exploit principles of natural neurons and peripheral nervous systems in a pragmatic engineering way, in particular, we currently use of spiking Integrate and Fire neurons and synapses to create inherently Self-x/Reconfigurable systems which can then evolve into self-adaptive systems to provide higher reliability and graceful degradation for future measurement systems. The target of our work is to achieve an adaptive signal conditioning system which will be benchmarked for instance with our 3D magnetic sensor for localization project [8].

In our previous work [16], we have shown that programmable Integrate and Fire neurons can be used with a Wheatstone half bridge to obtain a linear time coding, Using a full bridge this extends the approach to produce a linear redundant time difference coding. We have also shown using Monte-Carlo analysis that any deviations due to process variations can be corrected by adapting the synaptic weights. We have shown that this code can be converted to a 1-of$\mathrm{N}$ coding by based on Jeffress model of sound localization using coincidence detection [16]. We showed a 4 bit 1-of-N decoder using this technique. This technique is robust because the time or shape of the spike does not matter but it is only the presence or absence of the spike that is important. While this technique is effective in producing robust, programmable outputs which can be easily decoded by any microcontroller (Since only one neuron spikes per output), it is possible to extract more data using place coding. In our previous work, we introduced a basic place coding concept which reduces the area of the 4 bit 1-of-N decoder by half. In this work we will show that place coding can be used to obtain much higher resolution compared to the same circuit using coincidence detection technique shown in our previous work. 


\section{State of the Art}

Neural ADC circuits have been presented in various works from 1986 [2] [3] [4]. These circuits analog neurons which can provide fast approximate solutions and slower accurate solutions. While most of these circuits were able to provide robust, reconfigurable ADCs, they were still making use of amplitude coding. Time coded ADCs have also been presented in the last few decades. These include rate coded approaches similar to delta-sigma converters and Spiking neural ADCs.

There have been a few approaches in creating ADCs based on spiking neurons. The first approach while being mostly theoretical, created a concept for SNN ADC/DAC by making use of spike maps [13] [12]. This approach can be compared to Successive Approximation Converters in traditional analog electronics. The second work is from [6] in which the authors have created an extremely energy efficient 8bit ADC which works similar to a dual slope ADC. While these ADCs solve different problems, they do not make use of the advantages of spiking neural networks. They are not reconfigurable or programmable, The latter requires matched capacitors to obtain accurate information. Our goal is to make use of inherent advantages of neural networks to create a robust, programmable, adaptive sensory systems, in particular, for leading edge technologies [1].

\section{Neural Coding}

In our previous work [9], we discussed about the problem of neural coding. The main types of neural coding are rate coding, time coding and place coding. The difference between these codes has been explained in [15]. In rate coding, the information is encoded in the frequency of spike in a time window. While this approach is effective, it is too slow. In 1991 a paper [18], showed that it was not possible for rate coding to contain all information required for the functioning of the visual system of a Bowfly. The alternative "Time to first Spike" encoding was proposed. Since then various coding schemes have been studied by different groups to discover the codes used in nervous systems. Rank order coding was proposed in 1998 by Thorpe and Guartis [17]. In their work, 


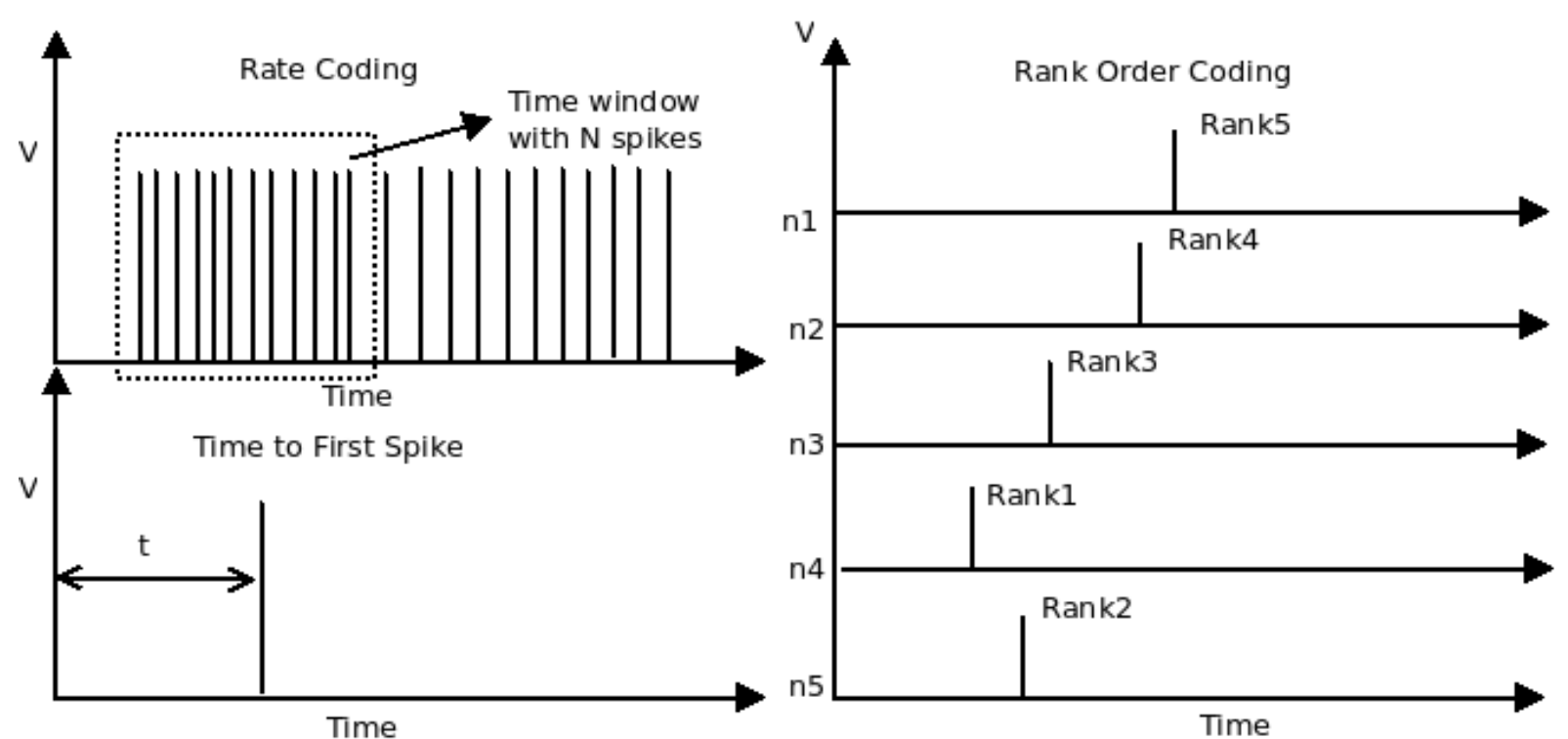

Figure 1: Neural Coding techniques

they describe Rank Order Coding used in cortical neurons for facial recognition [19]. The advantage of this coding over "Time to First Spike" codes is that there is no need for measuring the accurate time of the spike at all. Most of the information required can be obtained from a the order of the first spike in a population of neurons.

This method is faster that rate coding and more robust that Time to First Spike coding. This type of encoding can be very useful when creating robust ADCs with unreliable components.

\section{Analog VLSI Neuron}

There are many different models of spiking neurons like the Integrate and Fire model, Izhikevich model etc. The concepts proposed in our work should work with any neural models. We make use of Integrate and Fire model based on a neuron and synapse circuit by Indiver [11] which have been simplified for our work. We make use of both Integrate \& Fire(IF) and Leaky Integrate \& Fire neurons(LIF). M10 in Fig.2 is connected by a dotted line. This is because it is present only in LIF circuit. The neuron and synapse circuits have been described in our previous work [16]. We are working with austriamicrosystems $350 \mathrm{~nm}$ technology using Cadence 6 for our simulations. 

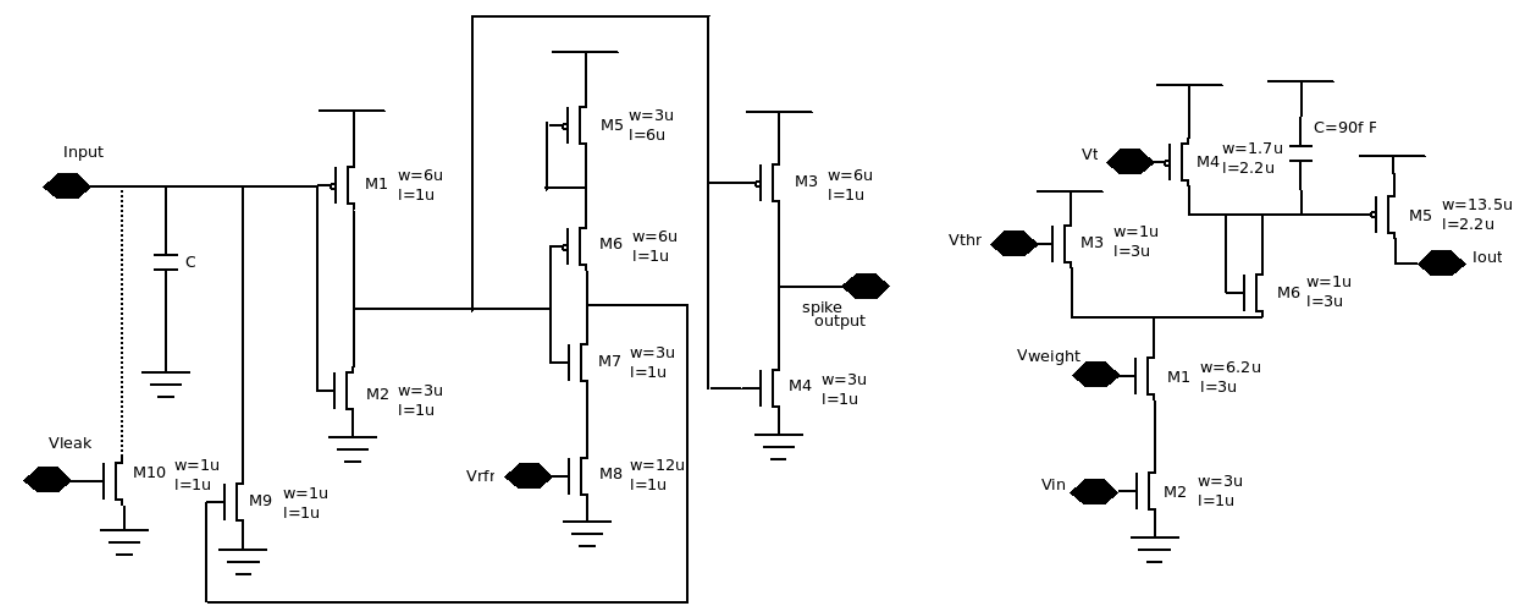

Figure 2: Integrate and Fire Neuron \& Synapse

\section{Differential Time Encoding}

When we discussed about neural codes, we were focused on Rank Order Coding to tap into its advantages. However, this code works better with an array of sensors. This is the reason behind its application mostly in image processing.

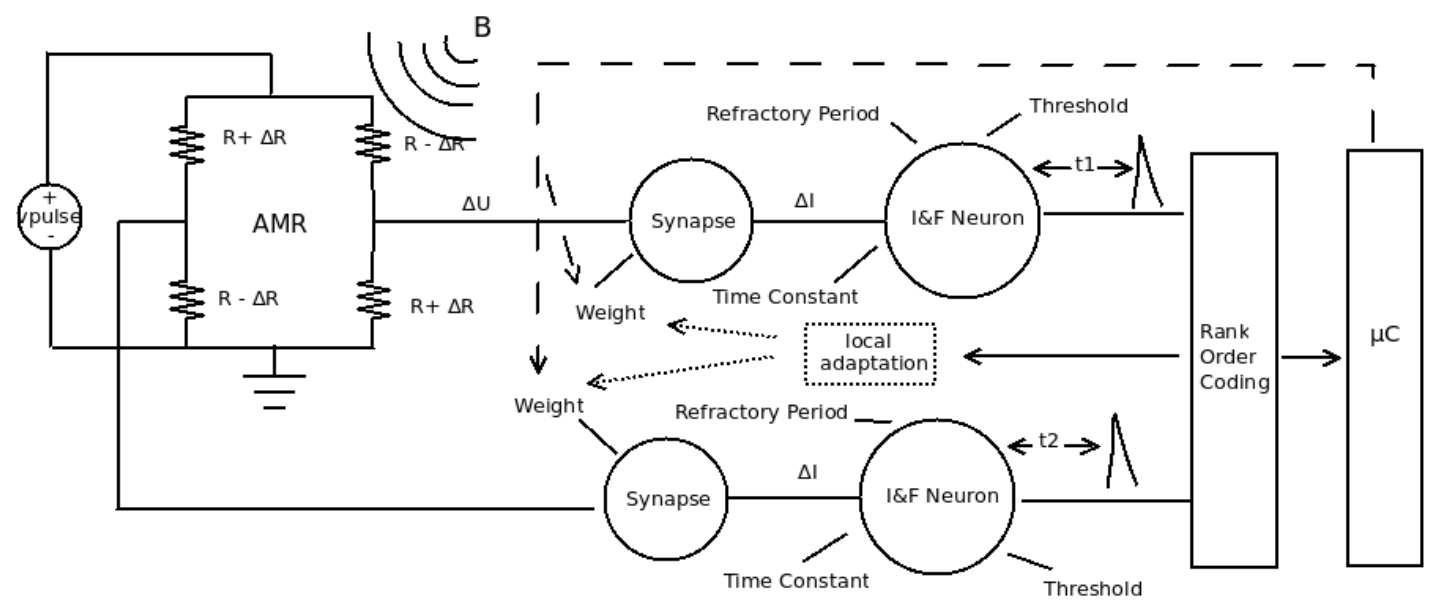

Figure 3: Transduction Principle

In this work, we are making use of an AMR sensor AFF755b [10] from Sensitec Gmbh. This sensor has a Wheatstone full bridge which could be considered as two 'sensors'. The IF neuron naturally encodes the amplitude signal into a Time-to-first-spike signal. Since the two halfs of the Wheatstone bridge act differentially, We obtain a differential time encoding. In our previous work, we have shown that this code is linear, we have also shown that process and temperature deviations can be corrected by changing the weight of the neuron . 


\section{Coincidence Detection \& One-of-N coding}

In 1948, a model was proposed for sound localization in mammals, making use of time delay neural network [14]. The ears implied in the model 4 can be easily replaced by our Wheatstone bridge. We are making use of the same model to convert the differential time data into a spatial code.

The VLSI IF neuron shown in Fig. 2 acts as a leaky IF when M10 is connected. We can use this circuit to create the coincidence detection neuron by having the $V_{\text {leak }}$ voltage at $1 \mathrm{~V}$. The $V_{\text {leak }}$ is actually creating a time based RBF function acting on the incoming spike. For a high $V_{\text {leak }}$ value $(1 \mathrm{~V})$ the radius of RBF is extremely small, and it becomes larger for lower $V_{\text {leak }}$ values.

In our previous work [16], we were able to create a 4.5 bit (21 levels) decoder by making use of $20 \operatorname{tabs}$ (10 on each side of the 'center') similar to Fig.4b, with each tab detecting one step. One of the benefits of coincidence detection is that it reduces the probability of error due to jitter since it depends on both the input spikes.

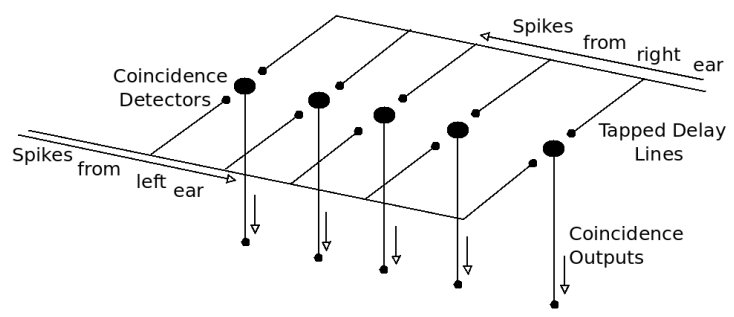

(a) Jeffress Model of Sound localization

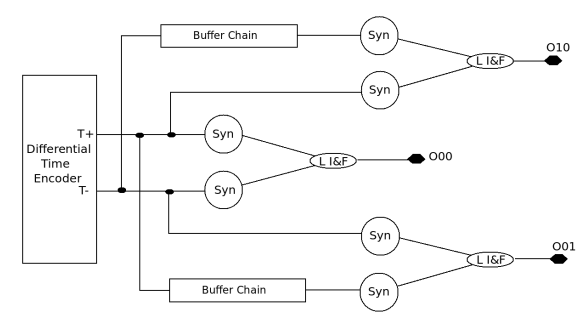

(b) Time Delay Neural Network Circuit with 2 delay lines

Figure 4: Coincidence Detection

\section{Place and Rank Order Coding}

In our coincidence detection technique, we made use of only one spike per input value. We create place coding by creating overlapping RBF kernels. We can do this when we reduce the $V_{\text {leak }}$ voltage to $0.6 \mathrm{~V}$ or $0.7 \mathrm{~V}$ as shown in Fig.5 which is similar to the Rank Order Coding shown in Fig. 1. In our current circuit, we are using buffer chains to produce delays as shown in Fig. 4b. This is just a temporary measure to analyse the place coding layer. We will be using neural delays in future similar to our previous work [16]. 

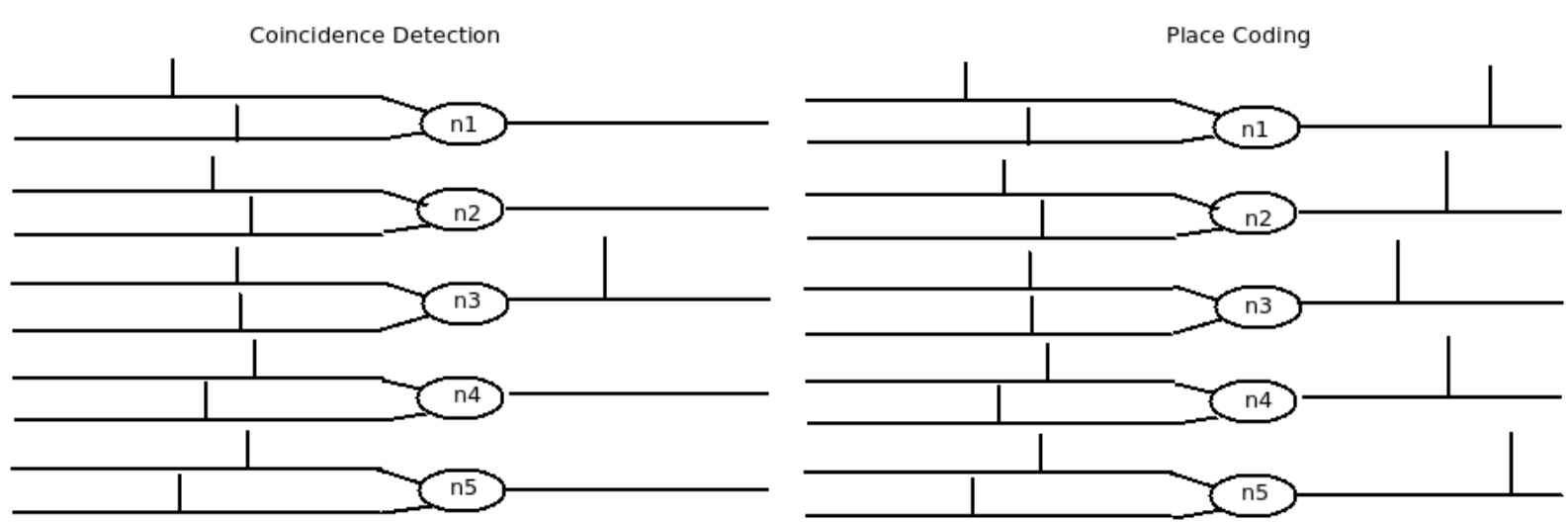

Figure 5: Coincidence Detection and Place Coding

We make use of Cadence Ocean scripts to run the simulations and provide the order of the spikes. This information is fed into python to analyse the data. In Fig.6, the rank order codes obtained are shown. The value "rvar" models the change in resistance of the AMR sensors in the Wheatstone bridge with regard to a simulated magnetic field influence [10]. We have 21 output neurons in our structure (similar to Fig.4b but symmetrically extended to 20 tabs.). The order of the first spike of neuron is used to create the place code list shown in Fig.6 assuming that the difference in spike times cannot be less than 0.5 nanoseconds. These results are sent for decoding.

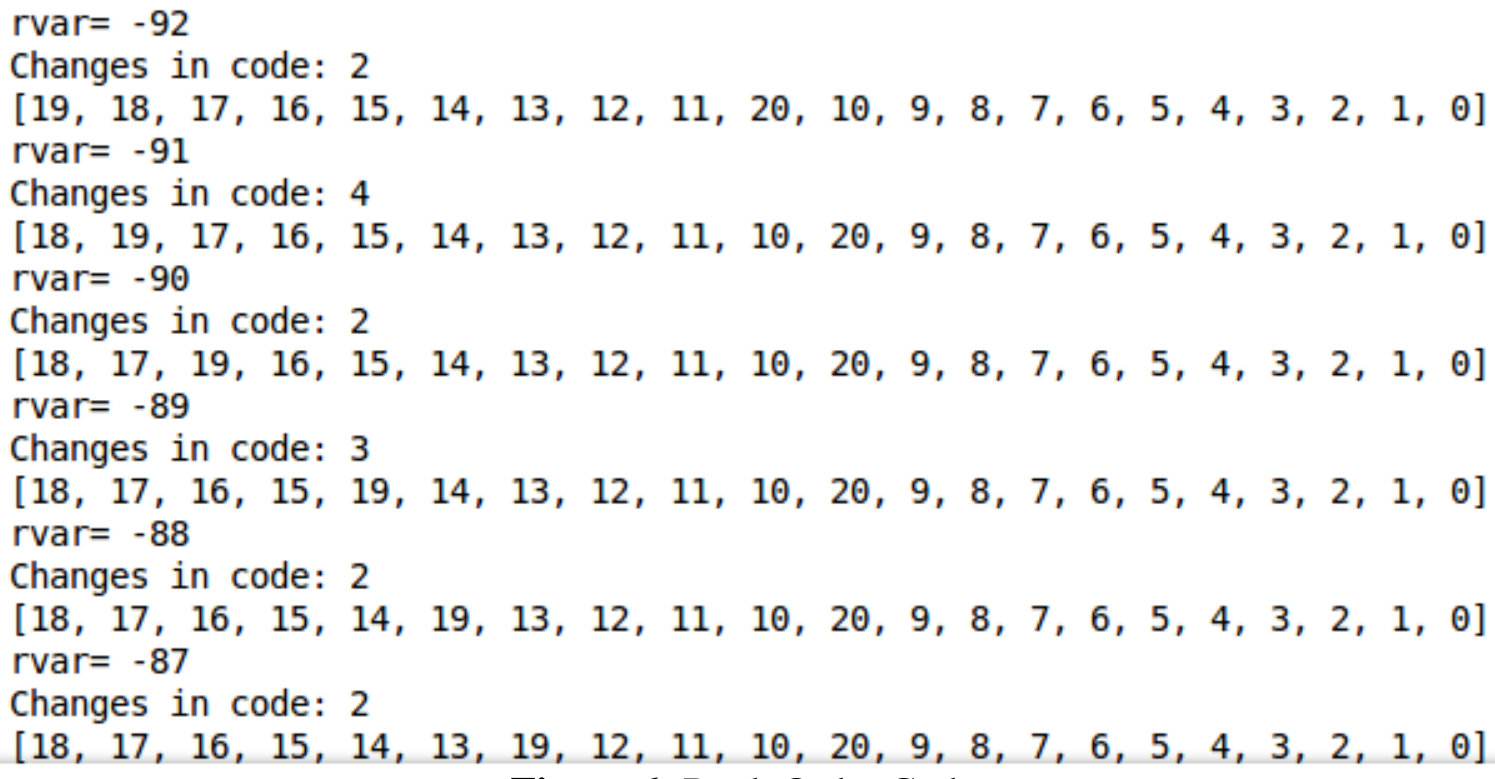

Figure 6: Rank Order Codes 


\section{Decoding}

There are several ways of decoding the place codes that we obtained. We introduced a rank order decoder in [9]. Such a decoder with modifications can be used for this purpose. In this work, we initially tried to make use of another supervised neural layer, which can be trained by a metaheuristic algorithm. Our goal was to make use of soft codes present because of time difference, which was not tapped by rank coding. This approach surprisingly is not yet competitive with a simple look-up table.

\subsection{Look-Up Table}

We created a decoder by using a simple look-up table in python. We converted the place codes shown in Fig. 6 into a base- 21 code. For instance, the place code shown for rvar $=-92 \Omega$ is 'jihgfedcbka9876543210', rvar $=-91 \Omega$ is 'ijhgfedcbak9876543210' and so on. This code is stored in a simple dictionary where a simple reverse search would provide the results.

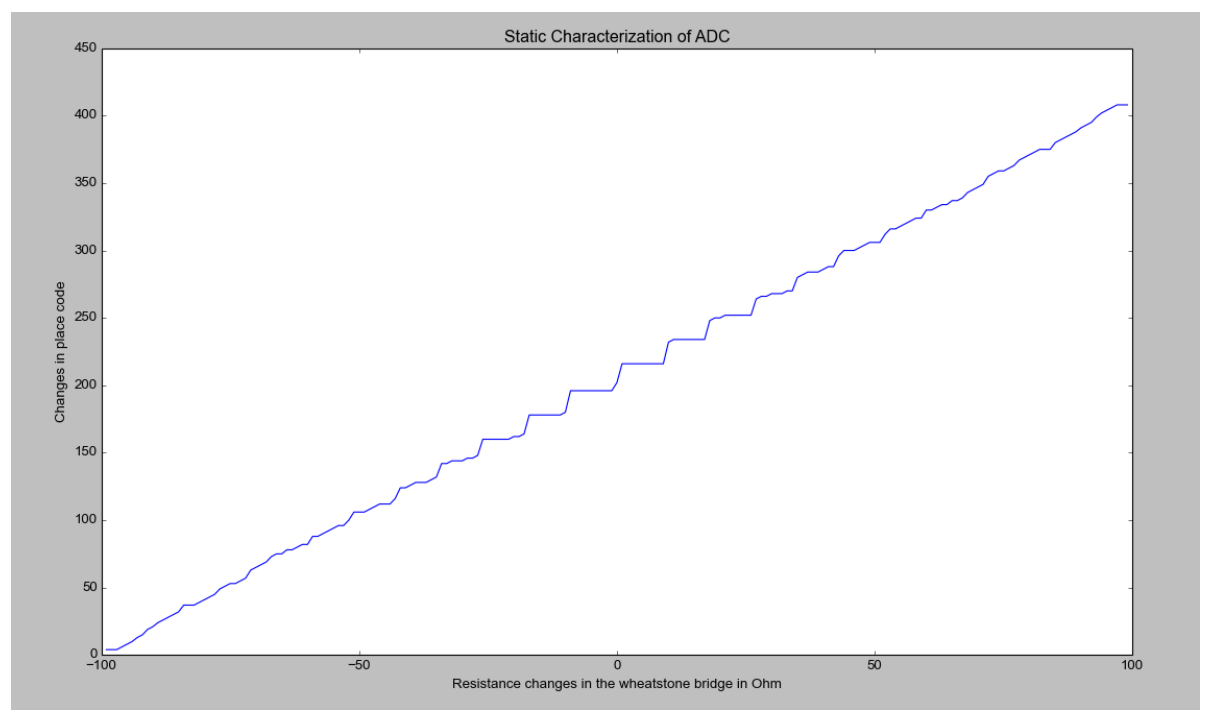

Figure 7: ADC characteristics

From the Fig.7 we can see that the ADC still is having a nonlinear,nonhomogeneous characteristic. Here the simulation was performed for $\Delta \mathrm{R}$ of $-100 \Omega$ to $100 \Omega$ in steps of $1 \Omega$. This can be translated to the values of particular sensor, i.e., $15 \Omega$ which corresponds to the $400 \mathrm{~A} / \mathrm{m}$ of the Sensitec AMR sensor [10]. The central region has a lower resolution while the fringes have a higher resolution. Currently, we are able to obtain a resolution of 6.3 bits (82 
levels). In the fringes, the ADC is able to resolve to $1 \Omega$ while it is able to resolve only to $7 \Omega$ in the center.

\section{Conclusion}

This work tried to contribute to the field of integrated sensor and measurement systems by a new concept of sensory signal conditioning and conversion. The goals are to achieve increased invulnerability to both device deficiencies, degradation, and faults met in aggressively scaled technologies as well as common noise, mismatch, and drift influences. This is tackled by employing concepts from neuromorphic engineering in a pragmatic approach. To meet the goals, we evolved a hierarchical spiking neuron architecture which transfers the sensory reading into pulse domain processing, employing differential time coding, coincidence detection, time delay structure, and rank order coding. Employing an AMR sensor as a research vehicle we modeled a Wheatstone fullbridge, read, and converted its output, achieving a principle resolution of 6.3 bits for $1 \mathrm{MHz}$ sampling rate. In the next steps of the work, we want to match the prototype to the exact span and possibly resolution of the commercial Sensitec AMR sensor AFF755B [10], increase resolution and linearity, and last but not he least include redundancy and adaptivity for neural delay and other parameters to achieve self-correction.

\section{References}

[1] ITRS Roadmap, http://public.itrs.net/

[2] Tank, D.; Hopfield, J.J., "Simple 'neural' optimization networks: An A/D converter, signal decision circuit, and a linear programming circuit," Circuits and Systems, IEEE Transactions on , vol.33, no.5, pp.533,541, May 1986

[3] Lee, B.W.; Sheu, B.J., "Design of a neural-based A/D converter using modified Hopfield network," IEEE J.of SSC , vol.24, no.4, pp.1129-1135,1989

[4] Avitabile, G.; Forti, M.; Manetti, S.; Marini, M., "On a class of nonsymmetrical neural networks with application to ADC," IEEE TCAS-I , vol.38, no.2, pp.202,209, Feb 1991

[5] Daponte, P.; Grimaldi, D.; Michaeli, L., "A full neural gray code based ADC," Instrumentation and Measurement Technology Conference, 1995. IMTC/95. 
Proceedings. Integrating Intelligent Instrumentation and Control., IEEE, vol., no., pp.795,, 24-26 April 1995

[6] Yang, H.Y.; Sarpeshkar, R., "A Bio-Inspired Ultra-Energy-Efficient Analogto-Digital Converter for Biomedical Applications ,TCAS-I , vol.53, no.11, pp.2349-2356, Nov. 2006.

[7] http://www.acam.de/de/produkte/picocap/measuring-method/.

[8] A. König ,D.Groben,A. C. Kammara and K. Thongpull, "3D-Localization of Low-Power Wireless Sensor Nodes Based on AMR Sensors in Industrial and AmI Applications", Proc. of 16 Int. Conf.SENSORS 2013, pp. 346-351.

[9] A.C. Kammara, J. Hornberger und A. König , Inherently Robust ADC Concepts with Biologically Inspired Spiking Neural Networks Rank Order Coding - A Case Study . In Tag. XXIV Messt. Symp. des AHMT, 23.-25. Sept., pp. 91-106, 2010.

[10] http://www.sensitec.com/upload/SENSITEC/PDF_Downloads/Datenblatt/ Sensitec_AFF755B_DSE_03.pdf.

[11] G. Indiveri: A VLSI Array of Low-Power Spiking Neurons and Bistable Synapses With Spike-Timing Dependent Plasticity, IEEE TNN, Vol. 17, No. 1, pp. 211-221, January 2006

[12] Takiguchi, Masao and Saito, Toshimichi,'Decoding Characteristics of D/A Converters Based on Spiking Neurons",Neural Information Processing, Vol. 5863, pp. 118-125, 2009,LNCS ,Springer Berlin Heidelberg

[13] Hamanaka, H.; Torikai, H.; Saito, T., "Spike position map with quantized state and its application to algorithmic A/D converter," Circuits and Systems, 2004. ISCAS '04. pp.673-678 Vol.4, 23-26 May 2004

[14] Peter Cariani (2011) Jeffress model. Scholarpedia, 6(7):2920.

[15] S. Thorpe, A. Delorme and R. Van Rullen: Spike-based strategies for rapid processing Neural Networks, Vol. 14, No. 6-7, 9 July 2001,pp. 715-725

[16] A.C. Kammara, A.König, Contributions to Integrated Adaptive Spike Coded Sensor Signal Conditioning and Digital Conversion in Neural Architecture, Sensoren und Messsysteme 17. ITG/GM Fachtagung, 3-4 Juni 2014

[17] Simon Thorpe, Jacques Gautrais, Rank Order Coding, Computational Neuroscience, 1998, pp 113-118

[18] W. Bialek, F. Rieke, R. R. de Ruyter van Steveninck and D Warland: Reading a Neural Code. University of California, Berkley, USA. Dpt. of Biophysics, Groningen, NL. Science Vol. 252, No. 28, pp. 1854-1857, 1991.

[19] R. VanRullen, R. Guyonneau and S. J. Thorpe: Spike time makes sense, Trends in Neurosciences, Vol. 28, Issue 1, Jan. 2005, pp. 1-4, 